\title{
Multi-target and Fuzzy Cloud computing Resource Scheduling Based on Intelligence Firefly Algorithm
}

\author{
Yu Zhang \\ School of information science and technology, Zheng Zhou normal university, \\ Henan, 45004 \\ E-Mail:20852192@qq.com
}

\begin{abstract}
According to jamming equation, the characteristics of array, this paper combined with jamming time-window, jamming efficiency ratio, jamming satisfaction, established multi-target fuzzy cloud computing jamming resource scheduling model with time-window, through the simulation, proved the scientificity and rationality of the method. The results showed, the improved firefly algorithm can preferably allocate the resources in cloud computing model, the effect of prediction model time is more close to actual time, can efficiently limit the possibility of falling into local convergence, the optimal solution's time of objective function value is shorten which meet the user's needs more.
\end{abstract}

Keywords: Multi-target, fuzzy cloud computing, Time-window, Resource scheduling, Firefly algorithm

\section{Introduction}

In the era of information war, electronic monitoring system gradually to digital, broadband, high power, high precision, interference integration of digitalizing broadband active phased array direction [1,2]. Such as the U.S. navy shipboard advanced multi-function radio frequency system (AMRFS), in which the function of radar, electronic monitoring and communication are integrated [3-5]. But now, the study on the integration of the system resource scheduling is less, this essay try to find an effective control method to solve the resource scheduling of electronic monitoring integrated system.

\section{Electronic Monitoring Integrated System}

Jamming power and the gain of antenna are related to factors such as the quantity of sub-array and the distance of sub-array, the jamming power $P_{j}$ and the gain of antenna $G_{j}$ are as follows:

$$
P_{j}=P_{s} N_{x} N_{y}
$$

(1)

$$
G_{j}=\frac{4 \pi \eta_{A} N_{x} d_{x} N_{y} d_{y} \cos \theta}{\lambda^{2}}
$$

In the formula, $P_{s}$ is the transmission power of each phased array unit. $\eta_{\mathrm{A}}$ is the efficiency of the antenna aperture. $N_{x}, N_{y}$ is the quantity of phased array units in horizontal and vertical direction respectively. $d_{x}, d_{y}$ is the interval of phased array 
units divided in horizontal and vertical direction respectively. $\theta$ is the angle between the target and the normal of sub-array. $\lambda$ is the wavelength of phased array radar [6].

If $d_{x}=d_{y}=\lambda / 2$, then formula (2) can be written as:

$$
G_{j}=\pi \eta_{A} N_{x} N_{y} \cos \theta
$$

(3)

According to the AMRFC, there are 1024 array units, which are double polarized and arranged by $32 \times 32$ in square. At first, array units are divided into 4 sub-array in 4 quadrant (quadrant of I , II, III, IV), again each quadrant (sub-array) is divided into 4 basic sub-array, which is consist of 64 fundamental array units in the phalanx of $8 \times 8$ [7].

Assuming that $P_{s}=4 w, \eta_{\mathrm{A}}=1, \theta$ is $0^{\circ}-45^{\circ}$, then we can calculate the power and the gain of different quantity of basic sub-array according to the formula (1) and (3), as shown in Figure1, Figure2.

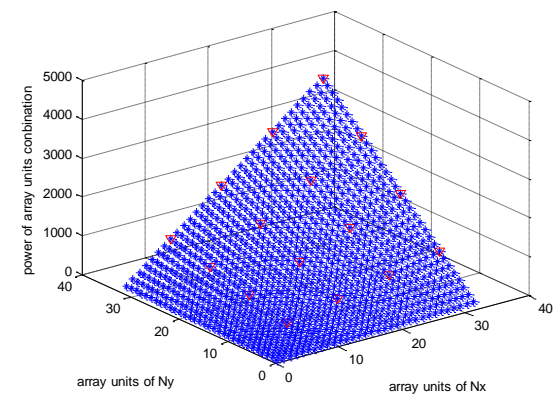

Figure 1. Power of Sub-Array

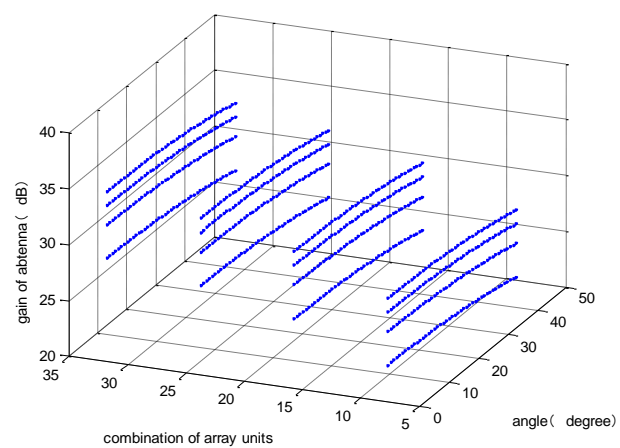

Figure 2. Antenna Gain of Sub-Array

\section{Analysis of Multi-target Interference}

Assuming that the target $i$ fly to ships straightly, the speed is $v_{i}$, the start time and distance of interfere is $T_{i s}$ and $R_{i s}$ respectively, the estimated end time and distance of interfere is $T_{i e}$ and $R_{i e}$ respectively. Target jamming time-window is $\left[T_{i s}, T_{i e}\right]$, namely the interference duration is $T_{i c x}$ :

$$
T_{i c x}=T_{i e}-T_{i s}
$$

$R_{i t}$ Is the distance between the target $i$ and the ship changed with the time $t$ as follows?

$$
R_{i t}=R_{i s}-v_{i} \times t
$$


$\left(N_{i x}, N_{i y}\right)_{t}$ is the quantity of array units needed at the time $t$ for interference to target $i$ :

$$
\left(N_{i x} N_{i y}\right)_{t}=\left(\frac{K_{i j} P_{i t} G_{i t} \sigma_{i}}{4 \pi\left(R_{i s}-v_{i} \times t\right)^{2}} \cdot \frac{L_{d}}{L_{j} \gamma_{j} K_{f}} \frac{1}{P_{s} \pi \eta_{A} \cos \theta_{i}}\right)^{1 / 2}
$$

Assume that each basic sub-array is composed of 64 array units, then the quantity of basic sub-array $Z_{i}(t)$ needed at the time $t$ for interference to target $i$ can be calculated as follows:

$$
Z_{i}(t)=\left\lceil\left(N_{i x}, N_{i y}\right)_{t} / 64\right\rceil
$$

$Z_{i}(t)$ Is positive (up) integer

Strategy of sub-arrays' allocation. Assume that the quantity of targets need to be interfered at the same time is $n, Z_{\max }$ is maximum interference power provided by array, the quantity of basic sub-array $Z_{i}(t)$ needed of multi-target interfered at the same time is:

$$
\begin{array}{r}
Z(t)=\sum_{i=1}^{n} Z_{i}(t) \\
\text { s.t. : } \quad Z(t) \leq Z_{\max }
\end{array}
$$

\subsection{Multi-target Scheduling Model}

Determination of resource scheduling decision stage The basis to determine resource scheduling decision stage mainly includes: (1) At the time when new target coming. (2) At the time when requirement of jamming resources increased to a basic sub-array. (3) At the time when resource competition fiercely needs to adjust interference target. (4) At the time when there are resources recycled.

Jamming Satisfaction, This paper defined the jamming satisfaction $\left({ }^{\alpha_{i}}\right)$ of target interference, it describes the fuzzy function between the actual time of interference and jamming time-window requested, as shown in formula (9):

$$
\alpha_{i}=\left\{\begin{array}{cc}
1 & T_{i c \bar{x}}=\Delta T_{i s j} \\
\left(\frac{\Delta T_{i s s} y^{z}}{T_{i c x}}\right. & T_{i c}>\Delta T_{i s}>0 \\
0 & \Delta T_{i s \bar{j}} 0
\end{array}\right.
$$

$\tau$ Is sensitive coefficient of the interference request for time?

Jamming Effectiveness Ratio As one of the bases of resource scheduling, jamming effectiveness ratio is $\beta_{i}$, it is the function of three indexes: expectations of target interference $^{E(i)}$, the resource using ratio $\frac{Z_{i}(t)}{Z_{\max }}$, and the threat grade of target $\omega_{i}$.

$$
\beta_{i}=\frac{E(i)}{\frac{Z_{i}(t)}{Z_{\max }} \cdot \omega_{i}}
$$

Objective Function of Resource Scheduling The goal of resource scheduling is to achieve the optimal value $E(\alpha)$ of jamming satisfaction of total interference targets, to get maximum value of jamming effectiveness $E(\beta)$ of limited resources. 


$$
\left\{\begin{array}{l}
E(\alpha)=\operatorname{opt}\left(\alpha_{1}, \alpha_{2}, \ldots, \alpha_{n}\right) \\
E(\beta)=\max \sum_{i=1}^{n} \beta_{i}
\end{array}\right.
$$

Principles of Resource Scheduling Set $W=\left[w_{1}, w_{2}, \ldots, w_{n}\right]$ represent the threat grade of targets, $\omega=1$ represent the highest threat grade, $\omega=2$ represent the second threat grade, $\omega=3$ and represent the lowest threat grade.

Principle 1: allocate resources to threat target if resource is enough and conditions allowed.

Principle 2: in order to achieve maximum interference efficiency, allocate resources to the threat target whose threat grade is higher, if resource is not enough and conditions allowed.

Principle 3: when resource competition occurs between the targets with the same threat grade, decision should be made according to the target jamming satisfaction and jamming effectiveness.

Principle 4: in order to keep the continuous of target jamming, decrease the times of array refactoring.

\section{Process of resource scheduling}

The process of jamming resource scheduling model as follows:

While time allows

Update the situation of resource using /recovery/remaining, and the jamming satisfaction of targets

determine whether need to allocate resource

While if need to allocate resource

determine whether the remaining resource can meet the demand

if the new resource demand is less than the resource remaining, then do resource allocating

else allocate or delay to allocate resource for the next stage, according to the value of $\omega, \alpha, \beta$

end if

determine whether need to do array refactoring

if need to do, then do array refactoring

end if

end while

\section{end while}

\subsection{Fuzzy Cloud Computing Resource Model}

The cloud model's fuzzy characteristic refers to the character of cloud drop distributes around cloud expectation curve's discrete degree. Professor Liuyu, etc. made researches on excess entropy measures cloud drop's discrete degree with fixed entropy. But these works did not show the essence factors of determine cloud model's fuzzy characteristic, $i, e$, the standard deviation Y's distribution of cloud drop quantitative data X determines the cloud model's fuzzy characteristic. The same as the cloud distribution probability density of cloud model algorithm identified is the theoretical basis of uncertainty reverse cloud model algorithm, this chapter revised the cloud distribution probability density and gave a strict proof according to fuzzy characteristic $\mathrm{Y}>0$. 
The positive direction cloud model algorithm steps in one-dimension theory's domain are as following:

Step 1: Generates normal random number $y_{i}$ whose expectation is $E_{n}$, standard deviation is He;

Step 2: Generates normal random number $x_{i}$ whose expectation is Ex, standard deviation is $y_{i}, x_{i}$ is a concrete and quantitative realize of qualitative concept $A$ operates in its corresponding quantitative theory of the domain $U$, called cloud drop qualitative data;

Step 3: Calculates $r_{i}=\exp \left(-\frac{\left(x_{i}-E_{x}\right)^{2}}{2 y_{i}^{2}}\right), r_{i}$ is the certainty degree or subjection degree of $x_{i}$ belongs to qualitative concept A;

Step 4: Repeats step one to three until generates $n$ cloud.

Prove: because $y-r\left(E_{n}, H_{e}^{2}\right)$, En refers to the discourse domain must be greater than zero, as $x \square N\left(E_{x}, y^{2}\right), \quad y$, as the standard deviation of $x$, must be greater than zero, so according to normal distribution random variable meets $3 \sigma$ rule, gets $E_{n} / H_{e} \geq 3$. Besides, the probability density of $\mathrm{Y}$ is

$$
x_{i}(y)=\frac{1}{\sqrt{2 \pi H_{e}}} \exp \left[-\frac{\left(t-E_{n}\right)}{2 H e^{2}}\right]
$$

When $x_{i}=y$, the conditional probability density is

$$
x_{i, j}(x \mid y)=\frac{1}{\sqrt{2 \pi y}} \exp \left[-\frac{\left(x-E_{x}\right)^{2}}{2 y^{2}}\right]
$$

Gets joint probability density through conditional probability density formula:

$$
x(i, j)=\frac{1}{2 \pi H_{e} j}-\exp \left[-\frac{\left(j-E_{n}\right)^{2}}{2 H e^{2}}-\frac{(i-E i)^{2}}{2 j^{2}}\right]
$$

Gets probability density which marginal probability density is cloud distribution through joint probability density formula:

$$
x_{i}(x)=\int_{x}^{y} \frac{1}{2 \pi H e y} \exp \left[-\frac{(y-E n)}{2 H e}-\frac{\left(x-E_{x}\right)}{2 y^{2}}\right]
$$

This formula has no analytic form Quod $x_{i}$ demonstrandum.

From step 2, 3, $y$ is the standard deviation of cloud drop qualitative data X, its distribution character directly determines the cloud drop's distribution character, the bigger distribution scale of Y, the more cloud drop distributes discrete. Because

$$
Y \sim N\left(E n, H e^{2}\right)
$$

This text takes $a=E n / H e$ as the measurement of cloud drop's discrete degree, called fuzzy factor, because qualitative data's standard deviation Y, En and He must be greater than zero at the same time so $a \geq 3$. Fuzzy factor $a$ integrative considers the nature that standard deviation $\mathrm{Y}$ of cloud drop's qualitative data $\mathrm{X}$ must be greater than zero, the distribution of $\mathrm{Y}$ directly affects cloud drop discrete degree and $a$ determines the distribution character of $\mathrm{Y}$, so 0.0 can be the significant digital characteristic of cloud model to presents the discrete condition of cloud drop's distribution. The fuzzy characteristic of cloud model has the following characters:

Character 1: The distribution characteristics of cloud drop's qualitative data standard deviation determines the cloud drop's distribution characteristics, a refers to the cloud drop's discrete degree and $a \geq 3$. The smaller $a$ be, the bigger discrete degree of cloud drop's distribution; when $\alpha=3$, the discrete degree of cloud drop's distribution reaches 
the biggest; the bigger $\alpha$ is, the smaller discrete degree of cloud drop's distribution, finally tends to normal distribution. Now the cloud drop all approximate distributes on cloud expectation curve.

Character 2: cloud distribution's corresponding range of fuzzy factor: $3 \leq a \leq 18$.

The fuzzy factor determines the distribution characters of cloud drop qualitative data, and the kurtosis describe the figure of data distribution at the same time, the kurtosis of normal distribution is 3 , if the kurtosis of cloud distribution values around 3, the cloud distribution turns to normal distribution[8]. The kurtosis of cloud distribution defines as following:

Definition 1: the kurtosis of cloud distribution

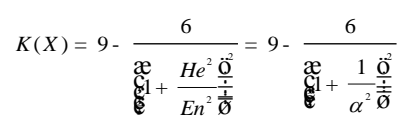

From this formula, fuzzy factor determines the transformation between cloud distribution and normal distribution. When $a=18$, the kurtosis of cloud distribution id 3.036, draws its cloud drop distribution as figure 3. From the figure, when cloud distribution approximate degrades into normal distribution. i,e, essentially, when fuzzy factor meets $3 \leq a \leq 18$, the distribution of cloud drop's qualitative data can be called as cloud distribution. So in later discussion, can only consider the condition when fuzzy factor meets $3 \leq a \leq 18$

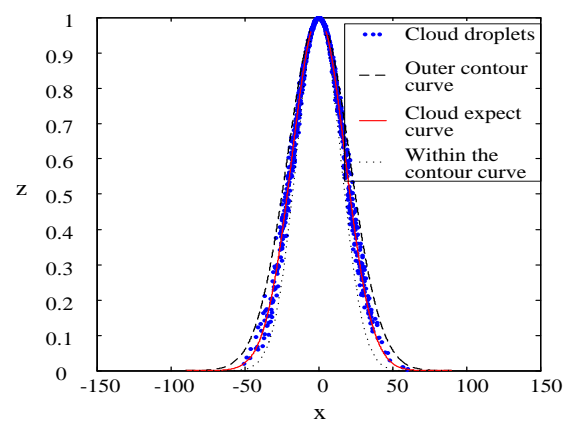

Figure 3. The Cloud Drop's Distribution Map When ${ }^{a}=18$

Character 3: From the distribution of $Y$, gets $99.7 \%$ cloud drop distributes between curve $^{f_{1}=\exp \left[\frac{-(x-E x)^{2}}{2(E n+3 H e)^{2}}\right]}$ and $f_{2}=\exp \left[\frac{-(x-E x)^{2}}{2(E n-3 H e)^{2}}\right]$.

F 1 is cloud mode's outer contour curve, $\mathrm{f} 2$ is inner contour curve.

The experiment result of cloud model's fuzzy characteristic is showed as figure 4. From this figure, the fuzzy factor of (a) is greater than (b), the cloud drop discrete degree of (a) is obviously smaller than (b). The fuzzy factor of (b) and (c) are being the same, their cloud drop discrete degrees are obviously being the same. The experiment showed, fuzzy factor can reflects the discrete degree of cloud drop's distribution, no matter how cloud model entropy and excess entropy values, if only their fuzzy factors are the same, the cloud model's cloud drop discrete degrees are the same. Besides, expectation (Ex) only affects the whole position of cloud drop's distribution without affects cloud drop's discrete degree. 


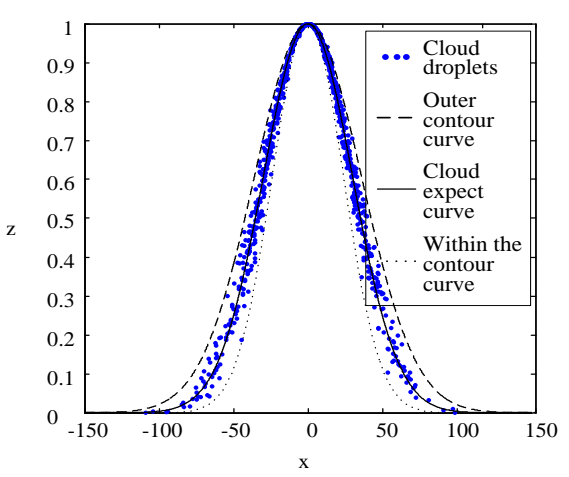

(a) $E x=0 E n=30 H e=2 \quad \alpha=15$

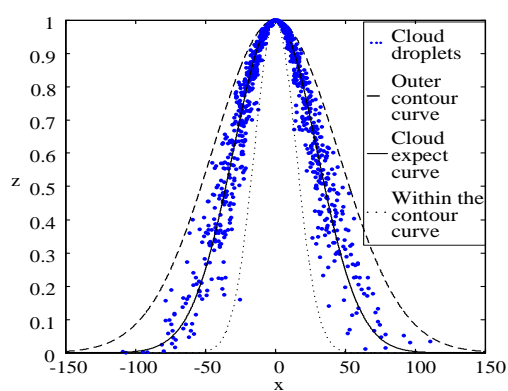

(b) $E x=0 \quad E n=30 H e=5 \alpha=6$

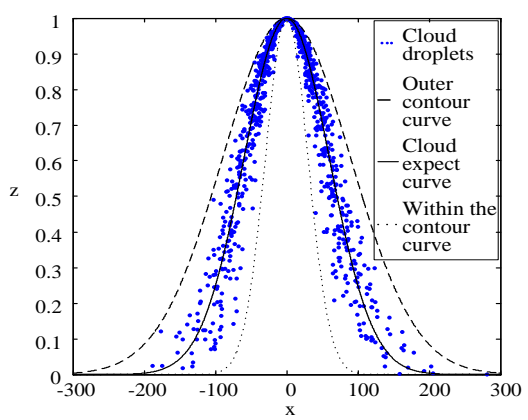

(c) $E x=0 E n=60 \mathrm{He}=10 \alpha=6$

\section{Figure 4. Cloud Drop's Discrete Degree Is Determined by Fuzzy Factor}

In cloud computing environment, the mostly used model is Map/Reduce; this model operates well in large-scale parallel task. Especially in cloud computing environment, it needs to processes each cloud user's resource number, time, network channel fee, etc. in time. The currently related task scheduling algorithm focuses on the needs of overall task, considers less about the cloud user's complementing time, which led to unreasonable in time and resources distribution for the users when multiple tasks operates. Supposes cloud client's tasks of cloud computing as table 1:

a) Divides large-scaled task into relatively small tasks, divides in average, the sub-tasks' operating time are similar.

b) The number of resource distribution offers enough for sub-tasks.

c) Reasonable defines sub-task occupies resources time. 
Table 1. Sub-Tasks and Resources Table

\begin{tabular}{|c|c|c|c|c|}
\hline $\begin{array}{l}\text { The } \\
\text { sub } \\
\text { tas } \\
\text { ks }\end{array}$ & $\begin{array}{l}\text { res } \\
\text { our } \\
\text { ces }\end{array}$ & $\begin{array}{l}\text { Running } \\
\text { time }\end{array}$ & $\begin{array}{l}\text { Running } \\
\text { costs }\end{array}$ & $\begin{array}{l}\text { Total } \\
\text { resources }\end{array}$ \\
\hline$n_{1}$ & $m_{1}$ & $t\left(n_{1}, m_{1}\right)$ & $\cos t\left(n_{1}, m_{1}\right)$ & \multirow{4}{*}{$S m=\sum_{i=1}^{n} m_{i}$} \\
\hline$n_{2}$ & $m_{2}$ & $t\left(n_{2}, m_{2}\right)$ & $\cos t\left(n_{2}, m_{2}\right)$ & \\
\hline $\begin{array}{l}\cdots \\
\cdots \\
\ldots\end{array}$ & $\begin{array}{l}\cdots \\
\cdots \\
\cdots\end{array}$ & & & \\
\hline$n_{n}$ & $m_{n}$ & $t\left(n_{i}, m_{j}\right)$ & $\cos t\left(n_{i}, m_{j}\right)$ & \\
\hline
\end{tabular}

$N_{i}$ Refers to the number of sub-tasks, $m_{i}$ refers to the number of resources, $t\left(n_{i}, m_{i}\right)$ refers to the time in task ${ }^{i}$, resources $j, \cos \left(n_{i}, m_{j}\right)$ refers to the costs in task ${ }^{i}$, resources $j$. In these above models, supposes the resources in cloud computing reasonable can be distributes into the computing resources of sub-tasks and ensures the shortest time and the lower costs for complementing the sub-tasks.

\section{Intelligence Firefly Algorithm}

intelligence firefly algorithm, proposed by KRISHNANAD, etc. in 2005, it's a new intelligence swarm optimal algorithm, this algorithm is wildly used in producing and scheduling, its simulated the search and optimize process to the firefly's attraction and migration, measured the advantages and disadvantages of the individual's position by solving the objective function. In this algorithm, each intelligence firefly distributes in the declaration space of objective function, this intelligence firefly has its field of view and carries fluorescent powder, the brightness of fireflies is related to its position and the fitness value of objective function, the brighter position shows the firefly there has pretty objective value and it can attract more fireflies to move towards this direction, as each firefly has its own range of view, the range can be affected by the neighbor fireflies, when the number of firefly becomes fewer, the range of view is larger and attracts more fireflies. When the fireflies are more, the range of view becomes smaller. But at last the position which most of the fireflies in is the optimal solution position.

Suppose the firefly swarm id N, the $i$ firefly's position $\left(x_{i}, y_{i}\right)$ matches the objective function $f\left(\left(x_{i}, y_{i}\right)\right)$ and the firefly's fluoresce in value is $T_{i}$, the updating formula of each firefly's range of view is:

$$
f_{k}^{i}(u+1)=\min \left\{f_{t}, \max \left\{0, f_{k}^{i}(u)+\beta\left(t_{u}-\left|t_{u}(u)\right|\right)\right\}\right\}
$$

Among them, $f_{k}^{i}(u+1)$ is the COOLEYE of $i$ firefly in $u+1$ range of view, $t_{u}$ is the threshold value of the neighbor firefly's number. $\beta$ is control constant, $t_{u}(u)$ is 
the number of firefly with high fluoresce in range of view. There, the formula of $t_{u}(u)$ is as following:

$$
t_{i}(u)=\left\{j:\left\|y_{j}(u)-y_{i}(u)\right\| \prec f_{k}^{i} l_{i}(t) \prec l_{j}(u)\right\}
$$

There, $y_{j}(u)$ is the position of $\mathrm{j}$ firefly in $\mathrm{t}$ generation, $l_{j}(u)$ is the $j$ firefly's fluoresce in value in $\mathrm{t}$ generation, the view of range between neighbor firefly is in $f_{k}^{i}$.

The firefly neighbor's selecting probability is:

$$
f_{i j}(u)=\frac{l_{i}(u)-l_{j}(u)}{\sum_{k \in n_{i}(u)} l_{k}(u)-l_{i}(u)}
$$

The position updating formula of firefly:

$$
f_{i}(u)=f_{i}(u-1)+s \frac{f_{j}(u-1)-f_{i}(u-1)}{\left\|f_{j}(u-1)-f_{i}(u-1)\right\|}
$$

Fluoresce in value's formula:

$$
f_{i}(u+1)=(1-t) l_{i}(u)+\gamma k\left(c_{i}(u+1)\right)
$$

In formula (16), $\gamma$ is a parameter to measure the function value, $k\left(c_{i}(u+1)\right)$ is the fitness value of the function.

In nature, supposes firefly $i$ enters into the view range of $j$, and the fluorescence in value is bigger than itself. Firefly $i$ chooses firefly $\mathrm{j}$ according to probability $t_{i j}(u)$, after choosing, the position of firefly $i$ and the fluorescence in is updating, calculates the objective function value of this position.

\subsection{Simulation and Calculation}

Parameter setting Assume there is 5 threat targets need to be interfered in a particular period, parameter settings as shown in table 2. In addition, set $\eta_{\mathrm{A}}=1, \frac{L_{d}}{L_{j} \gamma_{j} K_{f}}=6$, $\Delta t=3 s$ and $E(i)=1$ in simulation.

Table 2. Parameters Setting of Targets

\begin{tabular}{c|c|c|c|c|c|c|c|c|c}
\hline Target & $\begin{array}{c}P_{t} G_{t} \\
(\mathrm{KW})\end{array}$ & $\begin{array}{c}G_{t} \\
(\mathrm{~dB})\end{array}$ & $\begin{array}{c}R_{s} \\
(\mathrm{~km})\end{array}$ & $\begin{array}{c}v \\
(\mathrm{~m} / \mathrm{s})\end{array}$ & $\begin{array}{c}\theta \\
\left.{ }^{\circ}\right)\end{array}$ & $\begin{array}{c}T_{s} \\
(\mathrm{~s})\end{array}$ & $\begin{array}{r}T_{e} \\
(\mathrm{~s})\end{array}$ & $\begin{array}{c}K_{j \Sigma} \\
(\mathrm{dB})\end{array}$ & ${ }^{2}$ \\
\hline 1 & 5 & 36 & 18 & 300 & 0 & 1 & 21 & 5 & 1 \\
2 & 8 & 33 & 20 & 250 & 30 & 4 & 26 & 13 & 1 \\
3 & 13 & 31 & 15 & 400 & 10 & 8 & 26 & 5 & 1 \\
4 & 25 & 31 & 50 & 260 & 40 & 1 & 40 & 18 & 1 \\
5 & 20 & 34 & 50 & 250 & 20 & 1 & 45 & 14 & 1
\end{tabular}

Data analysis To demonstrate interference of resource scheduling in the case of insufficient resources, threat grade of all threat targets were set to 1 (highest grade), 
targets from the many different directions (the value of targets interval is 10 degree), time-windows of targets overlapped severely, the competition of limited jamming resources is fierce. Through simulation and calculation, the relationship between targets and array units demanded as shown in Fig 5, and the relationship between targets and the basic sub-arrays demanded as shown in Fig 6 .

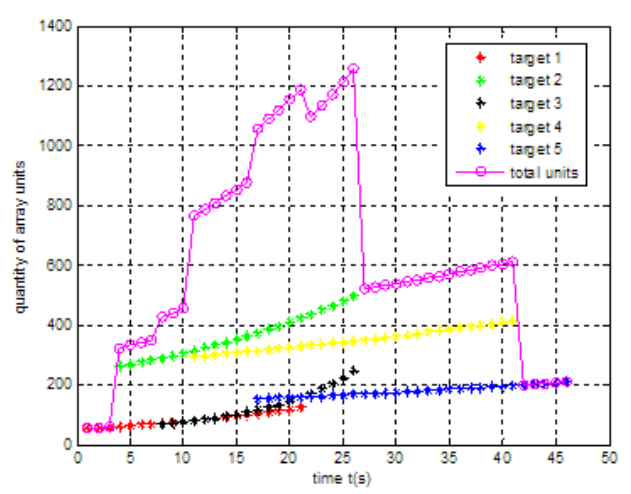

Figure 5. Relationship of Target and Units Demanded

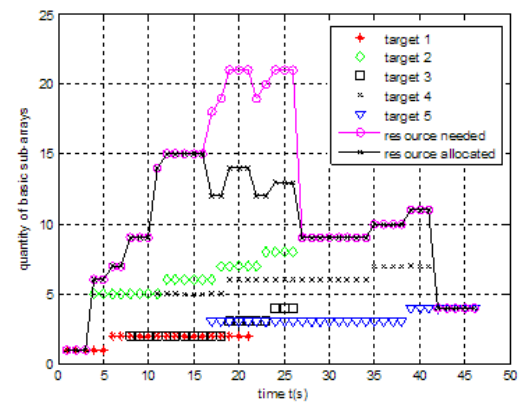

\section{Figure 6. Relationship of Target and Basic Sub-Array Demanded}

(1) Stages analysis of decision-making

In the simulation time, there are 15 points that need to make decisions of resource scheduling. At the points $(\mathrm{K}=1,2,4,5,7)$ are decision-making stages for 5 threat targets coming, namely are interference resource request moments of targets; At the points $(\mathrm{K}=3,6,8,10,12,13)$ are decision-making stages for threat target jamming resources required (basic sub-arrays) increased; At the points $(\mathrm{K}=9,11,14,15)$ are decision-making stages for target "disappear", and resource released.

(2) Analysis of array refactoring

At the point $\mathrm{K}=5$, target 4 began to request jamming resources ( 5 basic sub-arrays required), as shown in table 3 , there are 7 sub-arrays remaining undistributed at the stage $\mathrm{k}=4$, and there are 3 targets (target 1 , target 2, target 3 ) being interfered in total. Target 1 and 3 resources demand forecast were zero. Target 2 resources demand forecast need to add a basic sub-array in the next seconds. There are 5 basic sub-arrays remain in quadrant I and quadrant IV, if assign this 5 basic sub-arrays to target 4 at one-time, there will not basic sub-arrays for target 2 to extend in next decision-making stage, then have to do array refactoring once more and need to adjust jamming resources for target 1 and target 4 at the same time.

Therefore, at the point $\mathrm{k}=5$, in order to decrease the times of array refactoring and maintain continuous of interference, set a basic sub-array aside in quadrant IV for target 
2, do a array refactoring, adjust interference resources of target 1 from quadrant I to quadrant II, assigned remaining resources in quadrant I and quadrant IV to target 4.

At the point $\mathrm{k}=10$, after target 1 "disappeared" and release its resources, in order to keep the interference resources of target 3 and target 5 concentrated relatively, then do a array refactoring.

(3) Analysis of target interference cancelled

At the point $\mathrm{k}=7$, target 5 is coming and resource demand is 3 basic sub-arrays, 4 targets (target 1,2,3,4) are being interfered and occupied 15 basic sub-arrays, their jamming satisfaction value are 0.8, 0.54, 0.5, 0.54, jamming efficiency are 8, 2.7, 8, 2.7 respectively, and the resource extension of target $2,3,4$, is one basic sub-array estimate, so jamming resources of system is insufficient, it can not satisfy the interference of 5 targets at the same time.

Strategy 1: If interference of target 1 was cancelled by the principle of jamming satisfaction, 2 basic sub-arrays can be released, and interference of target 2 will be cancelled also to release resource for target 5. In this way, the sum of jamming satisfaction and jamming effectiveness of 5 targets is 4.39 and 23.2 respectively.

Strategy 2: If interference of target 2 was cancelled and release 6 basic sub-arrays, then don't need to cancel the interference of other targets in future, ensure the limited jamming resources interfere more targets and continuously. So, the interference of target 2 was cancelled at this decision-making stage to release resources for target 5 . The sum of jamming satisfaction and jamming effectiveness of 5 targets is 4.54 and 21 respectively.

Therefore, choose "strategy 2 " at the point $\mathrm{k}=7$, which is better than "strategy 1 ".

\section{Conclusion}

On the basis of giving out the resource allocate strategy of array, combined with jamming equation and target jamming time-window, for the problem of multi-target interference: the quantity of incoming target and the stages of interference are uncertain. This paper established jamming resource scheduling model based on multi-target fuzzy cloud computing with time-window. To allocate jamming resources of the electronic monitoring integrated system, according to two indexes, jamming satisfaction and jamming efficiency, to optimize resource scheduling with the maximum jamming satisfaction and the optimal jamming efficiency.

\section{Acknowledgement}

This work was supported by The Department of Henan Province (NO. D20141506)

\section{References}

[1] W. -W. Long, W. -X. Su, W. -F. Ding, et al, "Research on Radar Weapons function, China Radar", (2010) February, pp. 7-10.

[2] S. - H. Chen, "The development trend of the U.S. navy ship-borne electronic monitoring system", National defense science and technology, (2006), pp. 6-9.

[3] C. Wise, J. Armitage, "Electronic combat and weapon system integration, Journal of Electronic Defense", (1991) July, pp. 37-39.

[4] S. B. Herskovitz, "Electronic monitoring in the year 2000 and beyond", Microwave Journal, (1991) September, pp. 44-59.

[5] A. B. Michael, "Multifunction shared aperture systems or smart skins now", Journal of Electronic Defense, (1991) January, pp. 27-32.

[6] Q. -F Li and X. -J. Xu, "Research on Simulation Model of Phased Array Radar System", Journal of CAEIT, vol. 12, (1991) February, pp. 239-243.

[7] X. - C. Xiao, "New Development of Electronic Resources Multi-complexity, Multifunction RF Systems", Communication Countermeasures, (2007) April, pp. 3-7.

[8] S. Zhou, G. Aggarwal and R. Chellapa, "Appearance characterization of linear lamebrain object, generalized photometric stereo and illumination- Invariant face recognition", IEEE Trans on PAMI, vol. 29, no. 2, (2007), pp. 230-245. 
[9] A. Ali-Eldin, J. Tordsson and E. Elmroth, "An Adaptive hybrid elasticity controller for cloud infrastructures", IEEE Network Operations and Management Symposium, (2012), pp. 204-212.

[10] D. Breitgand, "A Epstein. SLA-aware Placement of Multi-Virtual Machine Elastic Services in Compute Clouds", IFIP/IEEE International Symposium on Integrated Network Management. Dublin, Ireland, (2011).

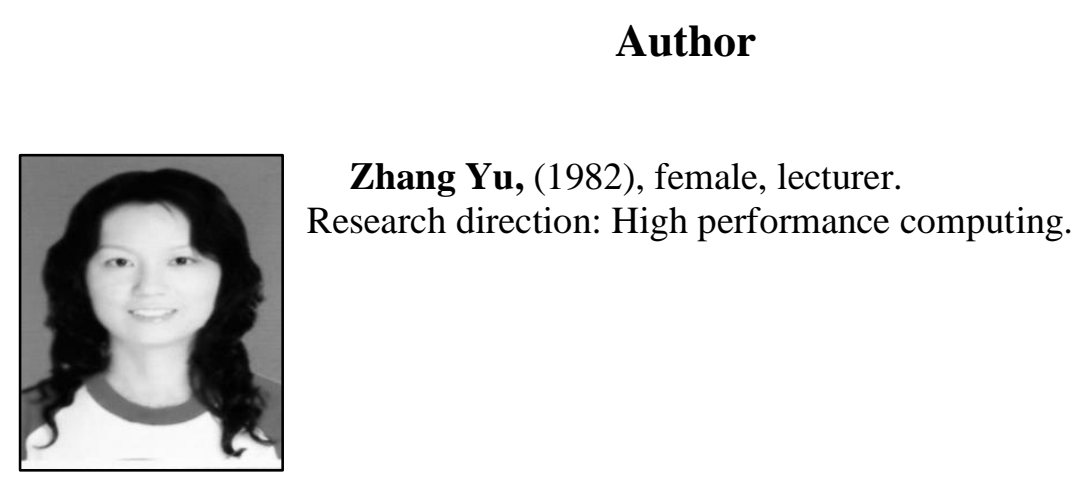

